The ASTROPHYSICAL JoURNAL, 371:432-442, 1991 April 10

(C) 1991. The American Astronomical Society. All rights reserved. Printed in U.S.A.

\title{
ABLATION OF SILICATE PARTICLES IN HIGH-SPEED CONTINUUM AND TRANSITION FLOW WITH APPLICATION TO THE COLLECTION OF INTERPLANETARY DUST PARTICLES
}

\author{
Aaron J. Rulison \\ Mechanical Engineering Department, California Institute of Technology 138-78, Pasadena, CA 91125 \\ RichaRd C. FlagaN \\ Chemical Engineering Department, California Institute of Technology 138-78, Pasadena, CA 91125 \\ AND \\ Thomas J. Ahrens and Wayne F. Miller \\ Lindhurst Laboratory of Experimental Geophysics, Seismological Laboratory, \\ California Institute of Technology 252-21, Pasadena, CA 91125 \\ Received 1990 May 25 ; accepted 1990 September 26
}

\begin{abstract}
A model for the ablation and deceleration of spheres in continuum and slip flow is presented. Experiments were conducted in which initially spherical 7.1 micron diameter soda-lime glass particles were launched from vacuum at $\sim 4500 \mathrm{~m} \mathrm{~s}^{-1}$ through a 0.5 mil $(13$ micron) plastic film into a capture chamber containing xenon at 0.1 and $0.2 \mathrm{~atm}$ and $295 \mathrm{~K}$. Samples of ablated particles were collected and inspected using scanning electron microscopy (SEM). It was found that the ratio of the ablated particle radius $\left(R_{f}\right)$ to the initial radius $\left(R_{0}\right)$ depends on the gas pressure such that at $0.1 \mathrm{~atm}, R_{f} / R_{0}=0.67 \pm 0.08$, and at $0.2 \mathrm{~atm}, R_{f} / R_{0}=0.88 \pm 0.08$. The model agrees with these results if the heat of ablation $Q$ is set to $1.5 \pm 0.2 \mathrm{MJ} \mathrm{kg}^{-1}$. This value of $Q$ approximately corresponds to the energy needed to raise the particle temperature from 295 to $1300 \mathrm{~K}$, the working point of soda-lime glass. This indicates that the mechanism of ablation is melting and blowing of material from the particle's surface.
\end{abstract}

Subject headings: interplanetary medium — laboratory spectra

\section{INTRODUCTION}

New instruments are needed to capture and analyze interplanetary dust particles (IDPs). Capture of the particles in a gas is attractive since the particle could be conveyed electrostatically to a planchet for detailed analysis (mass spectroscopy). An instrument using gas deceleration would have the gas confined within a thin plastic or metal membrane. High-speed $\left(10 \mathrm{~km} \mathrm{~s}^{-1}\right)$ dust particles would penetrate the membrane and then be stopped by gas drag and ablation. The gas cell must be large enough to accommodate the deceleration length of the incoming particles. The extent of ablation must be minimized if compositional analysis of single particles is to be undertaken. System mass and the mass of gas carried must be minimized for spaceborne missions. Gas pressure and temperature are design parameters that may be varied in the instrument optimization.

A detailed understanding of ablation processes and the aerodynamic forces acting on the particle is needed to design this instrument. Since the instrument will operate in the continuum and slip flow regimes, knowledge of ablation and deceleration is needed there. The ablative deceleration of particles entering an atmosphere at high velocity has been studied extensively to relate the radiant intensity and duration to the mass, density, and velocity of objects which intercept the Earth in order to constrain the contribution from different sources (e.g., comets and asteroids) (Becker \& Friichtenicht 1971; Becker \& Slattery 1973; Friichtenicht \& Becker 1971; Friichtenicht, Slattery, \& Tagliaferri 1968; Slattery \& Friichtenicht 1967). That work focuses primarily on ablation of particles in high-velocity free molecular flow, such as occurs in the atmosphere at high alti- tudes and is, therefore, not directly applicable for the design of this instrument.

This paper presents a study of ablative deceleration of particles in the continuum and slip flow regimes. The theory of ablative deceleration in free molecular flow (i.e., meteor theory) is reviewed and extended to the continuum and slip flow regimes. An experimental investigation of ablative deceleration is then reported and compared with meteor theory and the revised theory derived in this paper.

\section{ABLATION AND DECELERATION}

The deceleration and ablation of an object can be described by momentum and energy conservation equations:

$$
m \frac{d u_{1}}{d t}=-\frac{1}{2} \rho_{1} u_{1}^{2} C_{D} A_{p}
$$

where $m$ is the particle mass, $u_{1}$ is the free stream speed of the particle, $\rho_{1}$ is the free stream gas density, $C_{D}$ is the drag coefficient, $A_{p}$ is the particle projected area, and

$$
Q \frac{d m}{d t}=-\frac{1}{2} \rho_{1} u_{1}^{3} C_{E} A_{p},
$$

where $Q$ is the heat of ablation, and $C_{E}$ is the energy transfer coefficient. Note that even though equations (1) and (2) involve the free stream values $\rho_{1}$ and $u_{1}$ the values of $C_{D}$ and $C_{E}$ given below take into account the shock wave which is present due to the supersonic and hypersonic speeds.

The heat of ablation $Q$ is the energy per unit mass needed to remove material from the particle and eject it into the free 
stream. Implicit in equation (2) is the assumption that all of the energy reaching the particle is used to remove material (none is conducted into the interior of the particle). Thus, the time scale over which ablation occurs, $\tau_{a}$, is assumed to be no greater than the Fourier conduction time $\tau_{c}$. Thus we require

$$
\tau_{a}<\tau_{c} \sim R^{2} / D_{t},
$$

where $R$ is the particle radius and $D_{t}$ is the thermal diffusivity. If the assumption given by inequality (3) is not valid, then heat is absorbed throughout the particle body rather than concentrating at the surface, and equation (2) would not apply. For the particles examined in this paper, $\tau_{c}$ is approximately 6 microseconds. As will be seen below, the model incorporating this assumption predicts that most of the ablation occurs during the first few microseconds of flight. Therefore the assumption leads to a reasonable level of approximation.

In the meteor literature $C_{E}$ is usually called the "heat transfer coefficient." In this paper this term is reserved for the usual fluid mechanical quantity $h$,

$$
h=\frac{q}{A\left(T_{f}-T_{s}\right)},
$$

where $q / A$ is the heat per unit time per unit area transferred to a surface, $A$ is the surface area, $T_{s}$ is the temperature of the surface, and $T_{f}$ is the temperature of the fluid. In equation (4), $q$ is taken to be positive if heat flows into the particle surface. There is some question about the validity of this equation since it will be used for a very large temperature difference $T_{f}-T_{s}$. It will be used as a first approximation however.

If the particle is assumed to remain spherical throughout its entire flight, then

$$
m=\frac{4}{3} \pi R^{3} \rho_{p},
$$

where $R$ is the particle radius and $\rho_{p}$ is the particle density, and

$$
A_{p}=\pi R^{2} \text {. }
$$

Substituting equations (5) and (6) into equations (1) and (2) gives

$$
\frac{d u_{1}}{d t}=-\frac{3 \rho_{1} u_{1}^{2} C_{D}}{8 \rho_{p} R}
$$

and

$$
\frac{d R}{d t}=-\frac{\rho_{1} u_{1}^{3} C_{E}}{8 \rho_{p} Q},
$$

respectively. The particle's density, $\rho_{p}$, is assumed to remain constant throughout its flight.

Now we introduce the dimensionless variables $R^{\prime}=R / R_{0}$, $t^{\prime}=t v_{0} / R_{0}$, and $M_{1}^{\prime}=M_{1} / M_{10}=u_{1} / v_{0}$, where " 0 " subscripts indicate the state before ablation or deceleration begins and $v_{0}$ is the initial free stream speed. Thus equation (7) becomes

$$
R^{\prime} \frac{d M_{1}^{\prime}}{d t^{\prime}}=-\frac{3 \rho_{1} C_{D} M_{1}^{\prime 2}}{8 \rho_{p}}
$$

and equation (8) becomes

$$
\frac{d R^{\prime}}{d t^{\prime}}=-\frac{\rho_{1} v_{0}^{2} C_{E} M_{1}^{\prime 3}}{8 \rho_{p} Q} .
$$

The initial conditions for equations (9) and (10) are $R^{\prime}(0)=1$ and $M_{1}^{\prime}(0)=1$.
In order to solve equations (9) and (10), expressions are needed for $C_{D}$ and $C_{E}$. In meteor science it is commonly assumed that $\sigma=C_{E} / Q C_{D}=$ constant over the flight of the particle. The parameter $\sigma$ is known in meteor science (Bronshten 1983) as the "ablation parameter." If $\sigma$ is constant, then equations (9) and (10) may be integrated directly. Dividing equation (10) by equation (9) gives

$$
\frac{d R^{\prime}}{R^{\prime}}=\frac{1}{3} v_{0}^{2} \sigma M_{1}^{\prime} d M_{1}^{\prime} .
$$

Integrating over the flight of the particle,

$$
\begin{aligned}
\ln \left(\frac{R}{R_{0}}\right) & =\frac{1}{6} v_{0}^{2} \sigma\left(M_{1}^{\prime 2}-M_{1_{0}}^{\prime 2}\right) \\
& =\frac{1}{6} \sigma\left(u_{1}^{2}-v_{0}^{2}\right),
\end{aligned}
$$

or

$$
\frac{R}{R_{0}}=\exp \left[\frac{1}{6} \sigma\left(u_{1}^{2}-v_{0}^{2}\right)\right] .
$$

In general, the drag coefficient $C_{D}$ and the energy transfer coefficient $C_{E}$ are functions of the instantaneous Reynolds number, $\operatorname{Re}_{1}=2 \rho_{1} u_{1} R / \mu_{1}$, and Mach number, $M=u_{1} / a_{1}$, where $a_{1}$ is the speed of sound in the gas. Since the gas density $p_{1}$, and viscosity, $\mu_{1}$, and speed of sound depend on the pressure and temperature of the gas, $\sigma$ must be a function of pressure and temperature. Meteor theory gives no way of predicting the dependence, however.

A previously reported expression for $C_{D}$ and a derivation for $C_{E}$ will now be used to develop a more general description of the ablative deceleration of small particles. Forney, Walker, \& McGregor (1987) report a formula for $C_{D}$ as a function of $M_{1}$ and $\operatorname{Re}_{1}$. The expression is

$$
\begin{aligned}
C_{D}= & \left(C_{D_{0}}-2\right) \exp \left[-3.07 \gamma^{1 / 2} \frac{M_{1}}{\operatorname{Re}_{1}} g\left(\operatorname{Re}_{1}\right)\right] \\
& +\frac{h\left(M_{1}\right)}{\gamma^{1 / 2} M_{1}} \exp \left(-\frac{\operatorname{Re}_{1}}{2 M_{1}}\right)+2,
\end{aligned}
$$

where

$$
\begin{gathered}
C_{D_{0}}=\frac{24}{\operatorname{Re}_{1}}\left(1+0.158 \mathrm{Re}_{1}^{2 / 3}\right), \\
g=\exp \llbracket 2.88\left\{1+\tanh \left[0.33 \ln \left(\operatorname{Re}_{1}\right)-1.92\right]\right\} \rrbracket, \\
h=2.3+1.7\left(\frac{T_{s}}{T_{f}}\right)^{1 / 2}-2.3 \tanh \left(0.51 M_{1}\right),
\end{gathered}
$$

where $\gamma$ is the ratio of specific heats, $T_{s}$ is the particle surface temperature, and $T_{f}$ is the fluid temperature. Equation (14) includes theoretically predicted trends and also fits the available experimental data to which it was fitted to within plus or minus $10 \%$ for $10<\operatorname{Re}_{1}<1000$ and $M_{1}=3$ (Crowe 1967). This range of $\mathrm{Re}_{1}$ covers the present cases. The accuracy of $C_{D}$ at higher $M_{1}$ has not, however, been experimentally tested so the uncertainty is larger at higher Mach numbers.

Equation (14) includes effects of noncontinuum flow since any two of the three parameters $M_{1}, \mathrm{Re}_{1}$, and the Knudsen number $\mathrm{Kn}_{1}$, determine the third. The Knudsen number is the ratio of the gas mean free path, $\lambda$, to the particle radius and can 


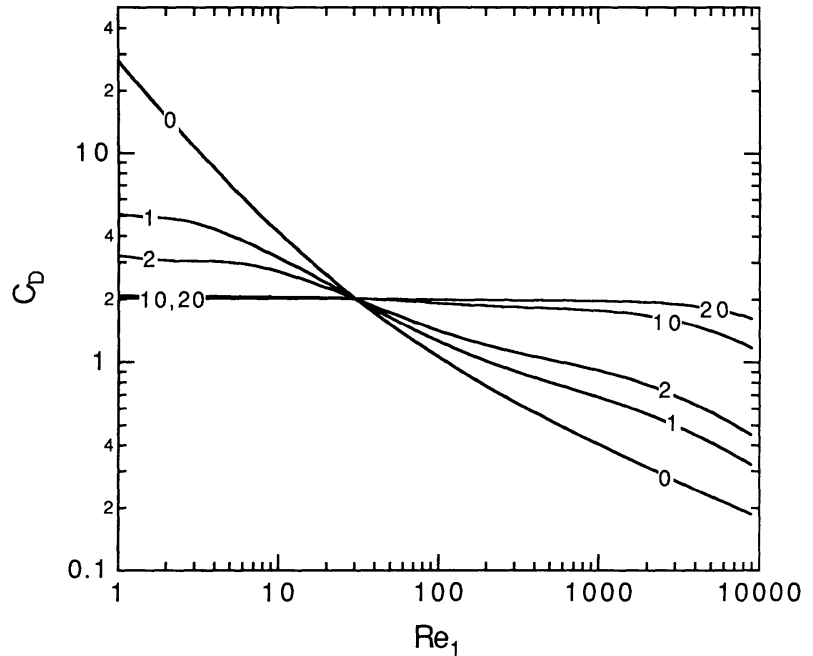
$M_{1}$.

Fig. 1.-Drag coefficient $C_{D}$ for a sphere as a function of $\operatorname{Re}_{1}$ for various

be expressed as (Eckert \& Drake 1972)

$$
\mathrm{Kn}_{1}=\left(\frac{\gamma \pi}{2}\right)^{1 / 2} \frac{M_{1}}{\operatorname{Re}_{1}}
$$

A flow is arbitrarily taken to behave as a continuum flow if $\mathrm{Kn}<0.01$, as a free molecular flow if $\mathrm{Kn}>10$, and as a transition flow if $0.01<\mathrm{Kn}<10$. Thus the flow is a continuum flow in the limit of $M_{1} / \operatorname{Re}_{1} \ll 1$ or a free-molecular flow in the limit $M_{1} / \operatorname{Re}_{1} \gg 1$. In the first limit (continuum flow) equations (14)-(17) give $C_{D}=C_{D_{0}}$. The second limit (free-molecular flow) to Equations $(14)-(17)$ yields $C_{D}=2$, the value that corresponds to reflections of incoming molecules off the sphere's surface. Figure 1 shows the variation of $C_{D}$ with $\operatorname{Re}_{1}$ for $M_{1}=$ $0,1,2,10$, and 20 . Sonic and supersonic flow reduce $C_{D}$ for $\mathrm{Re}_{1}<30$ and increase $C_{D}$ for $\mathrm{Re}_{1}>30$.

To derive an expression for $C_{E}$, he high-speed gas flow must be understood. In continuum and near-continuum flow, ablation occurs at the particle surface due to intense heating from the surrounding fluid. The fluid is hot because it has passed through a bow shock in front of the particle (Fig. 2a). The primary assumption of the present model is that the bow shock may be replaced by a normal shock for purposes of computing the fluid state surrounding the particle. This approximation is useful in that it greatly simplifies the problem of computing the heat transfer at the particle's surface which is a complicated phenomena involving boundary layer formation and the veloc-

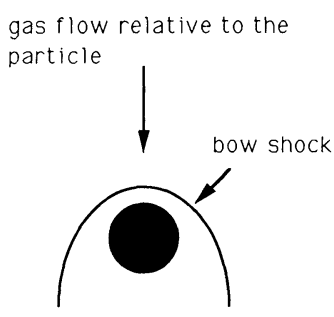

(a)

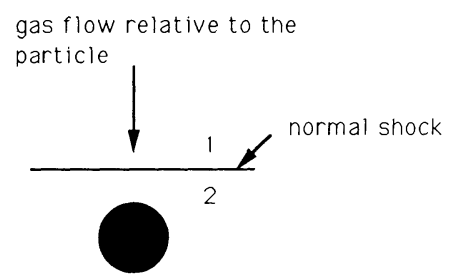

(b)
FIG. 2.-(a) Schematic diagram of the bow shock through which the gas must pass to reach the particle. (b) Approximation of the bow shock by a normal shock of the same Mach number. The fluid state upstream of the shock is denoted as 1 . The fluid state downstream of the shock is denoted as 2 . ity and temperature fields behind the curved bow shock. This approximation will not be valid if the fluid is too rarefied to form a shock. Thus use of this model is restricted to $0 \leq \mathrm{Kn} \leq$ $\mathrm{Kn}_{c r}$, where $\mathrm{Kn}_{c r}$ is the maximum $\mathrm{Kn}$ for formation of a bow shock. Bronshten (1983) gives $\mathrm{Kn}_{c r}=\frac{1}{3}$. The experimental conditions examined in this paper and the present IDP instrument satisfy this condition but meteor entry into the atmosphere may not. The fluid state behind the shock (region 2 in Fig. $2 b$ ) is thus uniquely determined by the normal shock relations and fluid state 1 ahead of the shock. For strong shocks the fluid can be expected to relax into its ionized state behind the shock after a sufficient number of collisions between its constituent molecules or atoms. This significantly modifies the state of the gas sufficiently far downstream of the shock. It is assumed here, however, that the fluid surrounding the particle has had insufficient time to relax and is therefore "frozen."

The heat flux to the particle is given by equation (2) as $\frac{1}{2} \rho_{1} u_{1}^{3} C_{E} A_{p}$. The same quantity can also be expressed as

$$
q=h_{2}\left(T_{2}-T_{s}\right) A,
$$

where $T_{s}$ is the particle surface temperature, $A$ is the particle total surface area, and $h_{2}$ is the heat transfer coefficient for the fluid in state 2 . By equating these we obtain

$$
C_{E}=\frac{4 h_{2}\left(T_{2}-T_{s}\right)}{(1 / 2) \rho u_{1}^{3}}
$$

The heat transfer coefficient, $h_{2}$, can be obtained from the surface-averaged Nusselt number $\mathrm{Nu}_{2}$ as

$$
h_{2}=\frac{\mathrm{Nu}_{2} k}{2 R_{p}} \text {, }
$$

where $k$ is the fluid thermal conductivity. In practice $k$ is evaluated at the average temperature of the fluid and particle surface.

To evaluate $\mathrm{Nu}_{2}$ the results of an analysis presented in Eckert \& Drake (1972) are used. If the fluid is rarefied, then the temperature of the particle surface will not exactly match that of the fluid at the surface. This temperature jump discontinuity modifies the continuum Nusselt number $\mathrm{Nu}_{20}$ such that

$$
\mathrm{Nu}_{2}=\mathrm{Nu}_{2_{0}} /\left[1+\left(\frac{\gamma \pi \theta^{2}}{2}\right)^{1 / 2} \frac{M_{2}}{\mathrm{Re}_{2} \operatorname{Pr}_{2}} \mathrm{Nu}_{2_{0}}\right],
$$

where

$$
\theta=1.996 \frac{2-\alpha_{s}}{\alpha_{s}} \frac{\gamma}{\gamma+1} \frac{1}{\operatorname{Pr}_{2}},
$$

$\operatorname{Pr}_{2}=\mu_{2} C_{p_{2}} / k_{2}$ is the Prandtl number of the gas as state 2, $C_{p 2}$ is the gas specific heat at constant pressure, and $\alpha_{s}$ is the thermal accommodation coefficient.

The continuum Nusselt number for a sphere surrounded by a laminar velocity distribution is (Eckert \& Drake 1972)

$$
\mathrm{Nu}_{20}=2+\frac{2}{\pi^{2}} \int_{0}^{\infty} \frac{\left[1+\exp \left(-\beta^{2} \pi\right)\right]\left(1+\beta^{4}\right)^{-1}}{\left[J_{1}^{2}\left(\alpha_{1} \beta\right)+Y_{1}^{2}\left(\alpha_{1} \beta\right)\right] \beta} d \beta
$$

where

$$
\alpha_{1}=\left(2 \operatorname{Re}_{2} \operatorname{Pr}_{2}\right)^{1 / 2}
$$

and $J_{1}\left(\alpha_{1} \beta\right)$ and $Y_{1}\left(\alpha_{1} \beta\right)$ are Bessel functions of the first and second kind.

Equation (24) can be integrated numerically for various $\alpha_{1}$ to 
obtain $\mathrm{Nu}_{20}$ as a function of $\alpha_{1}$. This integration was conducted for $3.65 \leq \alpha_{1} \leq 115$ which covers a wide enough range of $\mathrm{Re}_{2}$ and $\mathrm{Pr}_{2}$ for the present purpose. A curve was then fitted to the results which has a maximum deviation of $1.4 \%$ over this range of $\alpha_{1}$,

$$
\mathrm{Nu}_{20}=\sum_{i=1}^{10} \delta_{i} x^{i-1}
$$

where

$$
\begin{aligned}
x & =\log _{10}\left[\left(2 \operatorname{Re}_{2} \operatorname{Pr}_{2}\right)^{1 / 2}\right] \\
\delta_{1} & =2.19280 \\
\delta_{2} & =0.39345 \\
\delta_{3} & =0.50523 \\
\delta_{4} & =1.39274 \\
\delta_{5} & =0.60924 \\
\delta_{6} & =-1.48578 \\
\delta_{7} & =-0.06673 \\
\delta_{8} & =0.66629 \\
\delta_{9} & =-0.26443 \\
\delta_{10} & =0.03258
\end{aligned}
$$

Equation (26) is plotted in Figure 3 as a function of $\operatorname{Re}_{2}$ for $M_{2}=0.2,0.6$, and 1.0 for a monatomic gas and for $\alpha_{s}=0.8$. The lower limit of $M_{2}=0.2$ is used since it is the limiting downstream Mach number in a very strong shock in a monatomic gas. The reduction of $\mathrm{Nu}_{20}$ due to slip can be seen by considering increasing $M_{2}$ at a fixed $\operatorname{Re}_{2}$ on Figure 3 .

The viscosity $\mu_{2}$ is needed to calculate $\operatorname{Re}_{2}$. Kinetic theory gives an approximate expression (Hirschfelder, Curtis, \& Bird 1964),

$$
\mu_{2}=8.44 \times 10^{-25} \frac{\sqrt{M T_{2}}}{\sigma^{2}}
$$

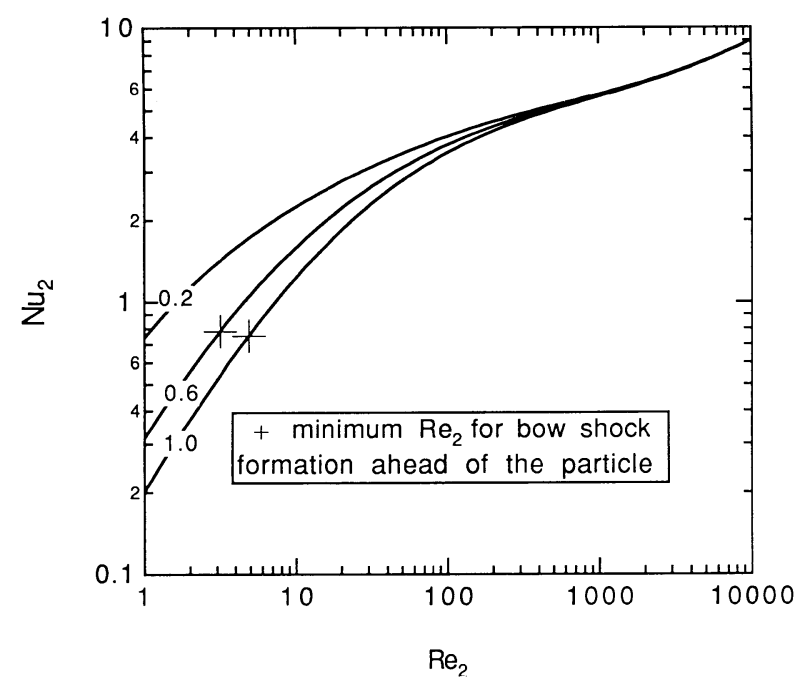

Fig. 3.-Nusselt number $\mathrm{Nu}_{2}$ for a sphere vs. $\mathrm{Re}_{2}$ for various $M_{2}$ and for $\operatorname{Pr}=\frac{2}{3}$ (monatomic gas). The minimum value of $\mathrm{Re}_{2}$ needed to satisfy the condition that a bow shock forms ahead of the particle is indicated by the crosses. This corresponds to the condition that $\mathrm{Kn}_{1} \leq 0.33$. In the $M_{2}=0.2$ case, the minimum $\operatorname{Re}_{2}$ is less than 1 . where $M$ is the molecular weight of the gas and $\sigma$ is the hard sphere collision diameter. The thermal conductivity $k_{2}$ is also obtained from kinetic theory as (Hirschfelder et al. 1964),

$$
k_{2}=2.632 \times 10^{-23}\left[\frac{\left(T_{2}+T_{s}\right)}{2 M}\right]^{1 / 2} / \sigma^{2}
$$

where both $\mu_{2}$ and $k_{2}$ are in mks units.

The Prandtl number

$$
\operatorname{Pr}_{2}=\frac{\mu_{2} C_{p_{2}}}{k_{2}}
$$

can be computed using equation (27), equation (28), and the following expression for $C_{p_{2}}$ :

$$
C_{p_{2}}=\frac{\gamma}{\gamma-1} R
$$

where $R$ is the gas constant.

In equation (30), it is assumed that $\gamma$ is constant; we consider this approximation reasonable for the present model. Note that for xenon, which was employed in the present experiments, $\gamma=5 / 3$. The gas temperature in the shocked state is calculated using the ideal gas, normal shock relation (Liepmann \& Roshko 1957),

$$
\frac{T_{2}}{T_{1}}=1+\frac{2(\gamma-1)}{(\gamma+1)^{2}} \frac{\gamma M_{1}^{2}+1}{M_{1}^{2}}\left(M_{1}^{2}-1\right),
$$

as is the pressure

$$
\frac{p_{2}}{p_{1}}=1+\frac{2 \gamma}{\gamma+1}\left(M_{1}^{2}-1\right) .
$$

The density then follows from the ideal gas law.

The velocity downstream of the normal shock is

$$
u_{2}=u_{1} \frac{\rho_{1}}{\rho_{2}}
$$

and the Mach number is

$$
M_{2}^{2}=\frac{1+[(\gamma-1) / 2] M_{1}^{2}}{\gamma M_{1}^{2}-[(\gamma-1) / 2]}
$$

Equation (9) and equation (10) can now be integrated numerically with the initial conditions $M_{1_{n=0}}^{\prime}=1$ and $R_{n=0}^{\prime}=1 . C_{D_{n}}$ and $C_{E_{n}}$ are evaluated from equation (14) and equation (26).

\section{EXPERIMENT}

Experiments were performed on soda-lime glass particles obtained from Duke Scientific Corp. A scanning electron micrograph of the particles as received shows that the particles are very nearly spherical (Fig. 4). Direct measurement of the diameters of the particles taken from several scanning electron micrographs gave the size distribution shown in Figure 5. In order to isolate the effects of varying sphere diameter on ablation, monosized particles would be desired. However, no attempt was made to size classify the particles in the original samples. The mean diameter was 7.1 microns. The nominal density was $2420 \mathrm{~kg} \mathrm{~m}^{-3}$.

The particles were launched using the Ames vertical gun range (AVGR) at NASA Ames Research Center, Moffet Field, California. AVGR consists of a light gas gun, launch tube, blast chamber, high-voltage spark gap velocity chamber, and vacuum impact chamber. In the light gas gun, a gunpowder 


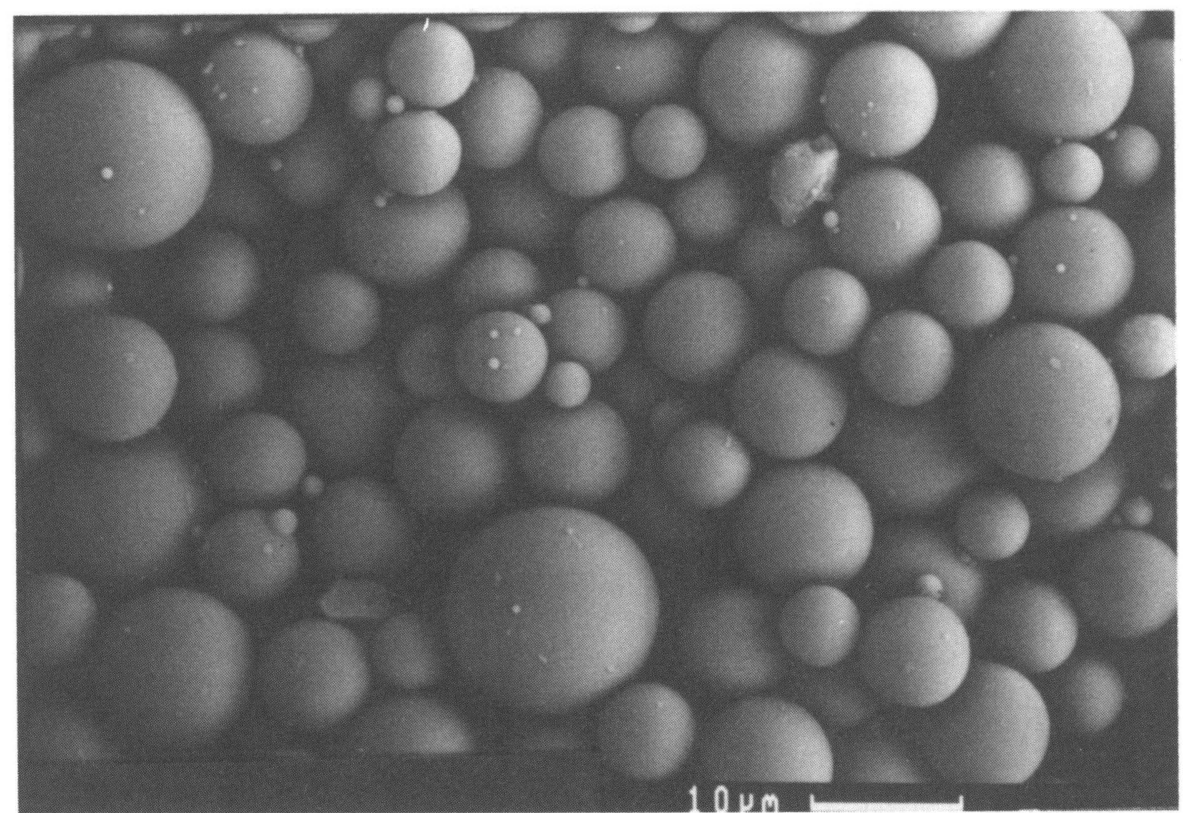

FIG. 4.-Scanning electron micrograph of the original (unablated) soda-lime glass spheres. The mean diameter is 7.1 microns.

charge is fired to drive a piston into a pump tube. The piston compresses hydrogen gas ahead of it into a high-pressure coupling. The hydrogen pressure and temperature increase until a diaphragm breaks, allowing the high-pressure hydrogen gas to accelerate the projectile down the launch tube toward the vacuum impact chamber. In this case the projectile consisted of an aluminum cup which held approximately $5 \mathrm{mg}$ of particles. An advantage of this particle launching system over systems which use electrostatic forces to accelerate the particles is its capability of launching relatively large particles. The cup is held in a plastic sabot, which fits in the gun barrel. The gun barrel is rifled so that the projectile assembly spins as it travels toward the impact vacuum tank. Centrifugal force breaks the sabot up and the pieces fly outward and are stopped by an impact sabot catcher before reaching the impact vacuum tank. Centrifugal force also forces some of the particles out and away

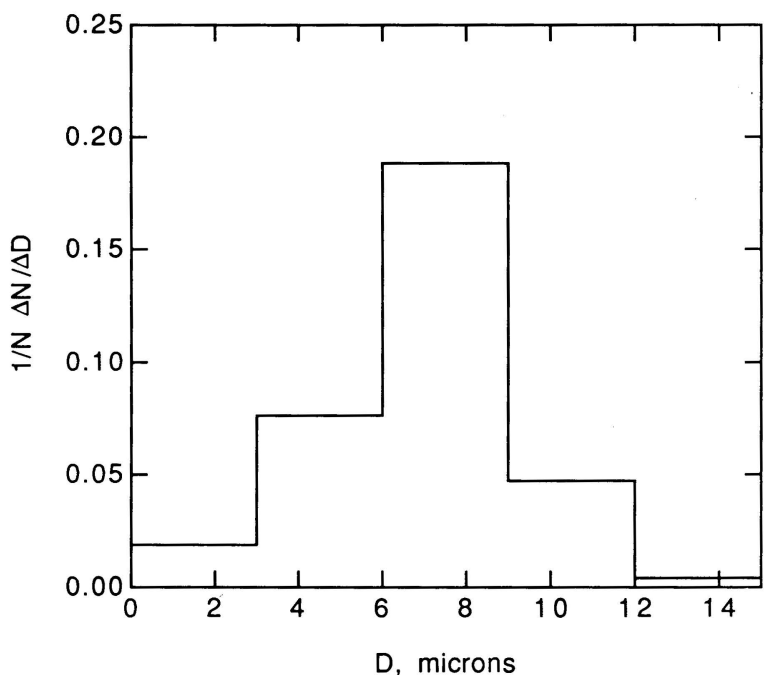

Fig. 5.-Size distribution of original (unablated) soda-lime glass spheres. The size distribution represents the diameters of 503 spheres measured by hand from SEM micrographs. from the cup. When the cup and particles reach the target, some of the particles are several centimeters away (radially) from the cup.

The target, shown in Figure 6, consisted of a xenon-filled Lucite capture chamber with a $10 \times 10 \mathrm{~cm}$ particle acceptance aperture, and $37 \mathrm{~cm}$ length. The center of the capture chamber has a $2.5 \mathrm{~cm}$ tube to allow the aluminum cup to pass without disturbing the experiment. Xenon is an appropriate gas for use in stopping IDPs because it is dense and chemically inert. Moreover, it is not an abundant constituent of IDPs, so it will not interfere with compositional analysis. A steel blast plate beneath the capture chamber stops the cup. The top of the capture chamber was sealed with $0.5 \mathrm{mil}$ (13 micron) Mylar or 0.5 mil cellulose triacetate film. The film was fastened to the capture chamber edges and central tube with contact cement. The capture chamber could hold $0.2 \mathrm{~atm}$ internal pressure (Mylar) or $0.5 \mathrm{~atm}$ (cellulose triacetate) before the film ruptured. The gas pressure was adjusted in the capture chamber before an experiment by valves outside the impact vacuum tank (Fig. 7). Valve 2 was left open and valve 1 was closed, while the impact vacuum tank was evacuated to $1 \mathrm{~mm} \mathrm{Hg}$ or

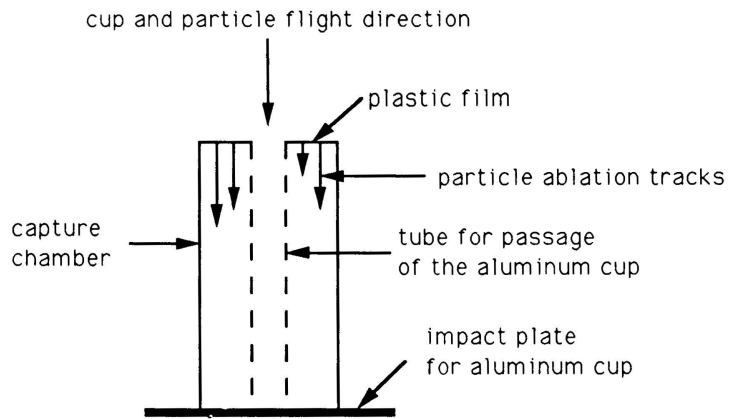

FIG. 6.-Schematic of the capture chamber used in the ablation experiments. The particles and aluminum cup move downward. The cup travels through the central tube while the particles penetrate the plastic film and enter the xenon gas. The particles settle to the bottom of the chamber to be collected on a Teflon sheet. The sheet was divided and mounted on electron microscope stubs. 


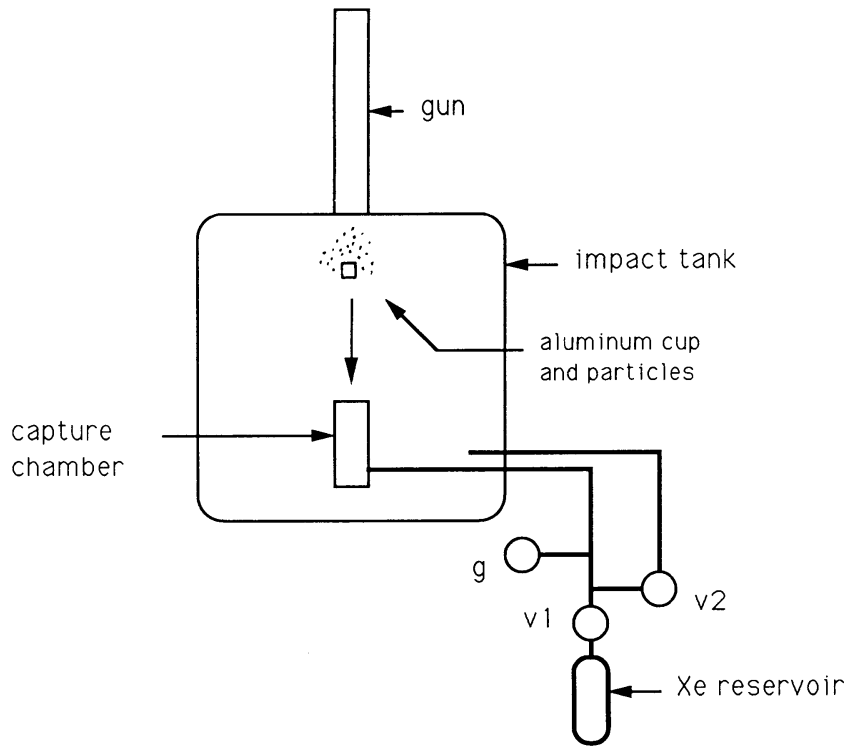

FIG. 7.-Schematic of the system used to adjust and maintain xenon gas pressure inside the capture chamber while the impact vacuum tank is evacuated. The gun is fired in the vertical position. The impact tank is evacuated to $\sim 1$ Torr.

less. Then valve 2 was closed and xenon was bled through valve 1 until the desired pressure was reached. Then all valves were closed and the gun was fired.

The bottom of the capture chamber was fitted with a Teflon sheet on which ablated particles settled after being stopped in the gas at the top of the chamber. After the experiment the Teflon was removed and divided into squares which fit on $13 \mathrm{~mm}$ electron microscope stubs.

The gun powder load in the powder chamber was designed to produce a projectile speed of approximately $5 \mathrm{~km} \mathrm{~s}^{-1}$. The actual projectile velocity was measured using the spark gap velocity chamber. Three shots each were conducted at 0.1 and $0.2 \mathrm{~atm}$.

A CamScan Series 2 scanning electron microscope fitted with a Tracor Northern TN5500 energy dispersive X-ray analyzer (EDS) was used to analyze the collected particles. Since gun blast debris also entered the capture chamber during a shot, it was necessary to use the EDS to verify that each measured particle was soda-lime glass. After a particle was positively identified as soda-lime glass, it was photographed. The particle diameter was determined using the equivalent area method.

In order to determine whether the particles were damaged as they penetrated the capture chamber films, particle capture tests were performed with the capture chamber evacuated so that no gas ablation could occur. Spheres launched through the film were subjected to the same SEM inspection as the ablated particles. No major damage is seen on these particles (Fig. 8). Thus, we conclude that any damage or reduction in diameter resulted from gas ablation. Nearly circular perforations in the film made by the particles are seen in Figure 9.

Only a small fraction of the original particles were thrown far enough from the projectile to penetrate the membrane rather than passing through the central tube. Particle deposition on surfaces other than the Teflon sheet further reduced the number of particles analyzed. Twenty-three particles were located, unambiguously identified, photographed, and sized in the 0.1 atmosphere experiment. The 0.2 atmosphere experiment yielded 22 particles. The average velocity of the projectile for the $0.1 \mathrm{~atm}$ case was $5000 \mathrm{~m} \mathrm{~s}^{-1}$ and for the $0.2 \mathrm{~atm}$ case was $4900 \mathrm{~m} \mathrm{~s}^{-1}$. The main reason so few particles were located is that only a small fraction of them were located far enough away radially from the cup to enter the chamber and not pass through the central tube.

\section{RESULTS}

The average diameter of the particles ablated at $0.1 \mathrm{~atm}$ was $4.7 \pm 0.6$ microns, and for 0.2 atm was $6.2 \pm 0.6$ microns. Views of two ablated particles are given in Figure 10. The initial size is shown for comparison. The ratios of the ablated radius to initial radius were $R_{f} / R_{0}=0.67 \pm 0.08$ and $0.88 \pm 0.08$ for 0.1 and $0.2 \mathrm{~atm}$, repectively. It is of interest to compare the prediction of the model described above with these results. The only parameter in the model which has not been determined is $Q$, the heat of ablation. There are two main mechanisms of ablation: vaporization and spraying of melted material. If the heats of fusion and vaporization of a material are $h_{f}$ and $h_{v}$ respectively, then the heat of ablation is roughly

$$
Q=h_{f}+\epsilon h_{v},
$$

where $\epsilon$ is the fraction of the mass that is lost by vaporization, $0 \leq \epsilon \leq 1$. Thus, $Q$ is restricted to $h_{f} \leq Q \leq h_{f}+h_{v}$. Equations (9) and (10) were solved numerically assuming $T_{a}=1300$ $\mathrm{K}$ (the working point of soda-lime glass; Bansal \& Doremus 1986 ) and $v_{0}=4500 \mathrm{~m} \mathrm{~s}^{-1}$ (the reduction in velocity from the measured projectile velocity approximates the deceleration of the particles through the film at the top of the capture chamber) for $p_{1}=0.1$ and $0.2 \mathrm{~atm}$. The working point of an amorphous material such as glass is defined to be the temperature at which the viscosity is $10^{4} \mathrm{P}$. It is the approximate temperature at which the glass may be formed. The curve of $R_{f} / R_{0}$ versus xenon gas pressure can be computed using various values of $Q$ (Fig. 11). It was found that a value of $Q=1.5 \pm 0.2 \mathrm{MJ} \mathrm{kg}^{-1}$ gives the results within experimental error for $R_{f} / R_{0}$ at both pressures. The curve for $Q=0.81 \mathrm{MJ}$ $\mathrm{kg}^{-1}$ does not extend below about $7 \times 10^{3} \mathrm{~Pa}$ because the requirement that $\mathrm{Kn}_{1} \leq 0.33$ would be violated during part of the particle's flight. Note that lower gas pressure leads to more ablation. This is mainly due to the fact that at lower pressures the particle is at speeds high enough to cause ablation for a longer time. Thus more material is removed.

We may now compare the experimentally determined value of $Q$ with the energy needed to raise the temperature of the soda-lime glass material from 295 to $1300 \mathrm{~K}$. An expression is available for the average heat capacity $C_{p}$ as a function of temperature for soda-lime glasses (Bansal \& Doremus 1986). At $1300 \mathrm{~K}$ the average heat capacity is $1.00 \times 10^{3} \mathrm{~J} \mathrm{~kg}^{-1} \mathrm{~K}^{-1}$ which gives $1.3 \mathrm{MJ} \mathrm{kg}^{-1}$. This roughly corresponds to the value of $Q=1.5 \pm 0.2 \mathrm{MJ} \mathrm{kg}^{-1}$, indicating that ablation occurs primarily by melting and blowing of material from the particle's surface. This indicates that the forces tending to resist ablation from the liquid phase (surface tension and viscosity) are overcome and vaporization is not required to remove material. This conclusion is reached if $T_{a}$ is greater than about $1100 \mathrm{~K}$. It is difficult to determine $T_{a}$ since this involves determining when the glass is soft enough (i.e., has low enough viscosity) that the aerodynamic forces can blow material from the particle's surface. For example, if $T_{a}$ is $1100 \mathrm{~K}$, then $Q$ is approximately $50 \%$ higher than the energy required to heat the particle from 295 to $1100 \mathrm{~K}$, and one would begin to think that 

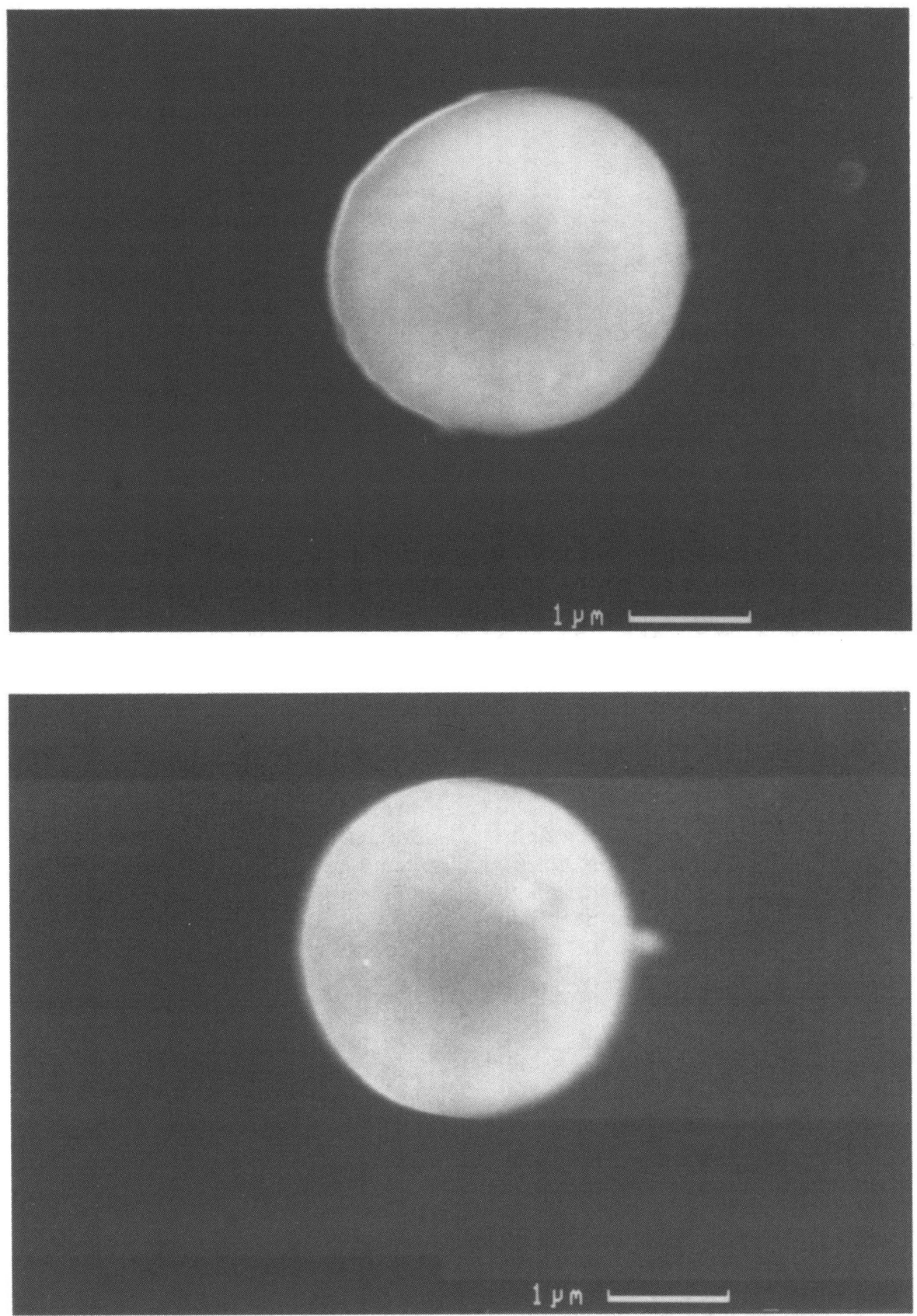

FIG. 8.-Typical scanning electron micrographs of particles which perforated the film but were not exposed to gas, so that no ablation could occur. The particles are undamaged, indicating that the reduction of diameter seen in Figure 10 must be due to gas ablation. The small white square seen on the micrographs is an aiming aid for the EDS system.

vaporization as an ablative mechanism is important in these experiments. Our best estimate for $T_{a}(=1300 \mathrm{~K})$ however, indicates that vaporization is unimportant.

Also indicated in Figure 11 is the best available result from meteor theory, which normally assumes $\sigma=C_{E} / Q C_{D}=$ constant. Clearly, meteor theory is inadequate to explain the results of these experiments.

The $\mathrm{Kn}_{1}$ number histories for typical conditions are shown in Figure 12. The $\mathrm{Kn}_{1}$ number increases as the particle ablates. Clearly, the lower pressure cases bring about higher $\mathrm{Kn}$ numbers as the particle is more extensively ablated.

The numerical simulations also show that $R_{f} / R_{0}$ is dependent on $R_{0}$ and $v_{0}$ (Fig. 13). Smaller and faster spheres are ablated more. Again, the assumption that $\sigma=$ constant gives no dependence on $R_{0}$. The curves are terminated on the lefthand side where the restriction that $\mathrm{Kn}_{1} \leq 0.33$ would be violated.

The time-resolved motion and ablation are computed for the two pressures used in the experiments (Fig. 14). At $0.1 \mathrm{~atm}$ it takes approximately 22 microseconds to slow the sphere to the speed where it no longer ablates. At 0.2 atm it takes 13 microseconds. The reason that more ablation occurs at 0.1 atm is that the sphere is decelerated more slowly, thus exposing it to high fluid temperatures for a longer time, allowing more material to be removed. Most of the ablation occurs during the first several microseconds for both 0.1 and $0.2 \mathrm{~atm}$. The measured extent of ablation agrees with the calculated final radii at both pressures within experimental error. 

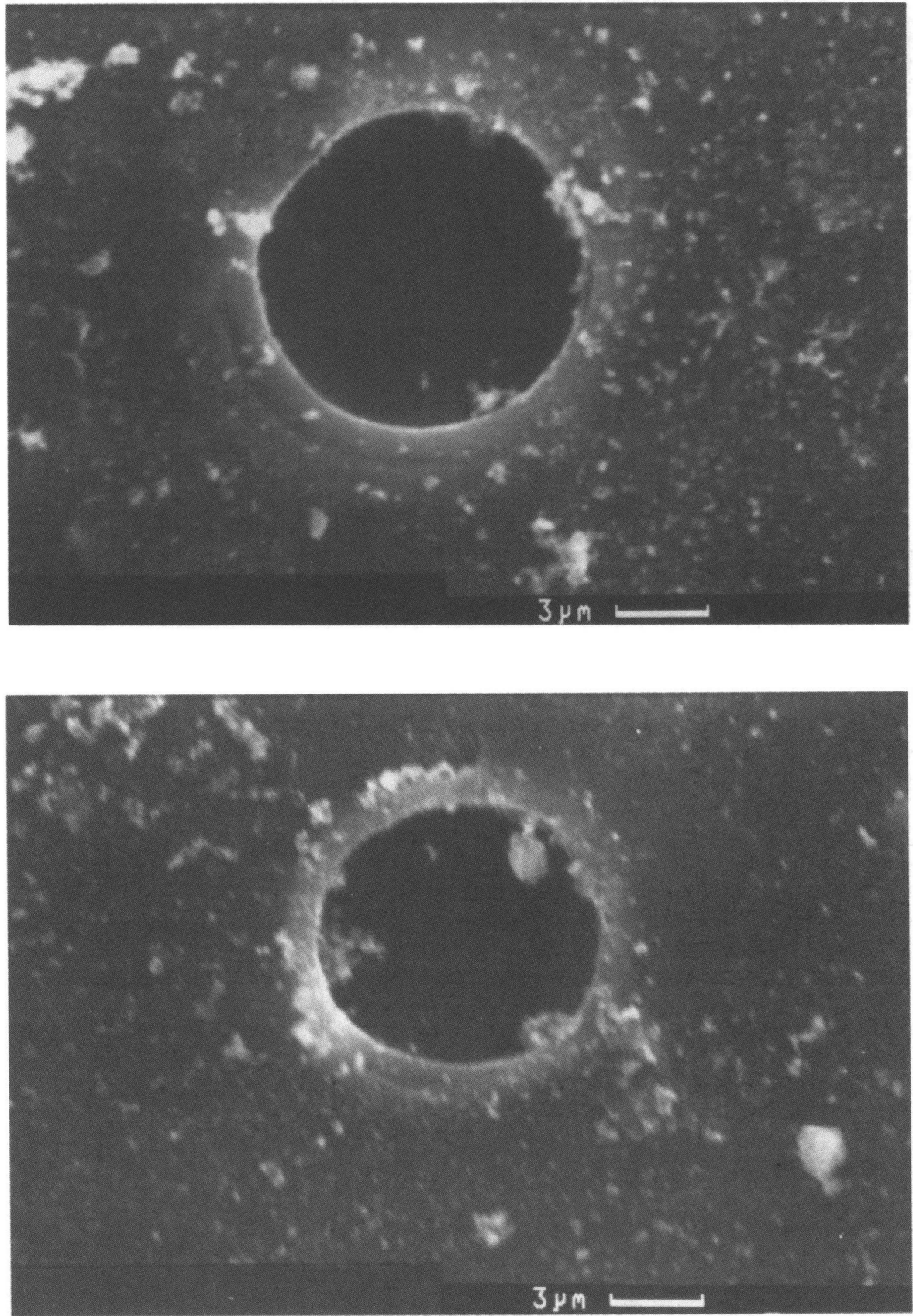

FIG. 9.-Typical scanning electron micrographs of film perforations made by high-speed particles. The film is 13 microns thick. The debris seen on the film is from the gun blast, and is not related to the glass particles.

Since various mineralogies of IDPs are expected to be encountered in space, the dependence of $R_{f} / R_{0}$ on the particle density and heat of ablation has been investigated (Fig. 15). As $\rho_{p}$ increases, $R_{f} / R_{0}$ decreases. Also, particles with greater heats of ablation are not as extensively ablated.

Because of the difficulty of the experimental method only two data points are given. The model presented here is therefore not strongly tested. There are many assumptions and simplifications discussed in the derivation. To improve the model would require a more detailed analysis of the gas flow and temperature fields around the particle as well as an analysis of the temperature and material phase fields within the particle. The variaton of ablated radius with gas pressure is brought out, however, and this is a step forward.

\section{CONCLUSIONS}

A model has been developed to describe the ablation and deceleration of spheres in continuum and slip flow. The model accounts for the variation of the particle drag coefficient and energy transfer coefficient with Reynolds and Mach numbers. The mass loss by ablation is pressure-dependent according to this theory. Experiments performed using the NASA Ames vertical gun range show the predicted dependence. The ratio of final to initial radii was $0.88+0.08$ for deceleration of silicate particles of 3.5 micron initial radius in $0.2 \mathrm{~atm} \mathrm{Xe}$ gas, and decreased to $0.67 \pm 0.08$ in $0.1 \mathrm{~atm}$ gas. The mass loss increases with decreasing pressure mainly because the reduced particle drag leads to longer exposure to high temperaures. The heat of 


\section{RULISON ET AL.}

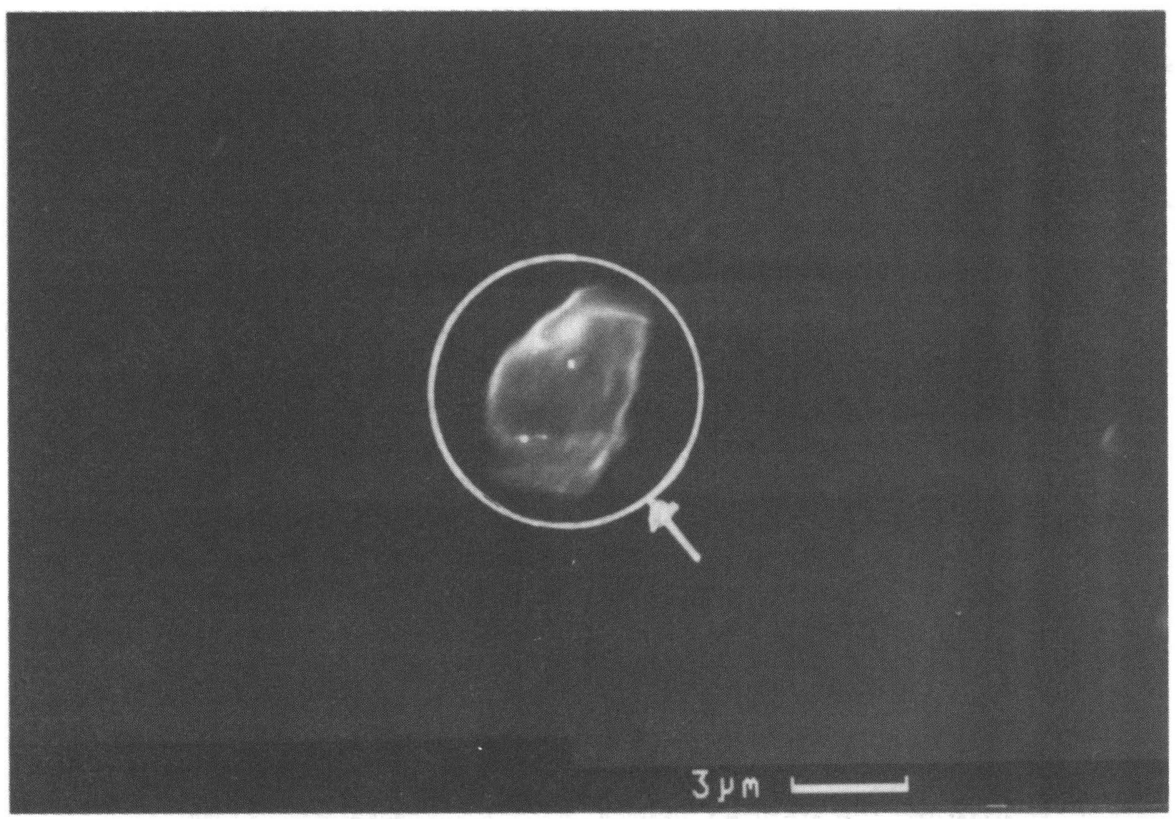

FIG. $10 a$

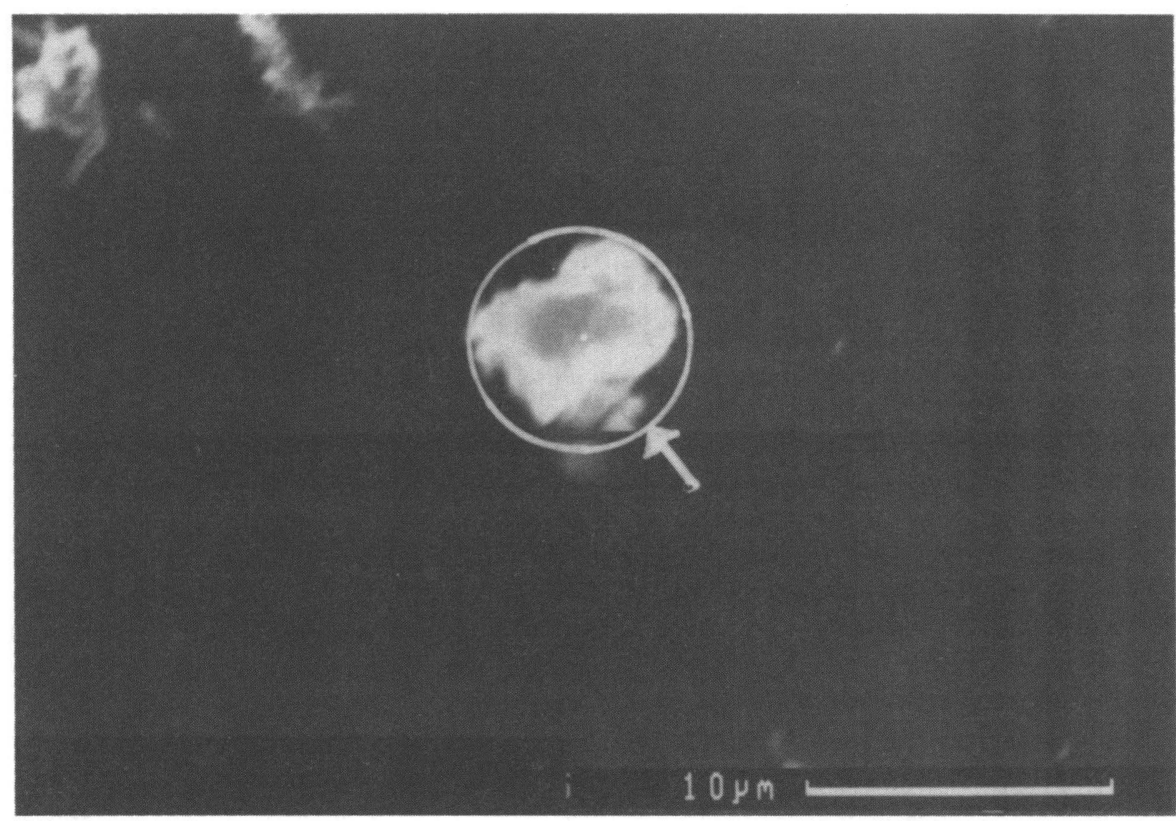

FIG. $10 b$

FIG. 10.-Scanning electron micrographs of soda-lime glass particles ablated in (a) $0.1 \mathrm{~atm}$ and (b) $0.2 \mathrm{~atm} \mathrm{Xe}$ gas at $295 \mathrm{~K}$. The initial speed was approximately $4500 \mathrm{~m} \mathrm{~s}^{-1}$. The initial sizes (arrowed), calculated for these particles by the average ratios of final to initial radius for each pressure, are indicated for comparison.

ablation was estimated to be $1.5 \pm 0.2 \mathrm{MJ} \mathrm{kg}^{-1}$, approximately corresponding to the energy required to raise the particle temperature from 295 to $1300 \mathrm{~K}$, the working point of the glass. This heat of ablation suggests that the primary mechanism of mass loss is aerodynamic entrainment of molten material from the surface of the article, and that vaporization plays only a minor role in ablation under the present experimental conditions. Parametric studies of ablation using the model suggest that the mass loss will increase with an increase in particle density, velocity, and radius, mainly due to the increased time required to slow the particle, or with decreasing energy of ablation.

We thank W. Logsden, B. Langedyk, and J. Vongraey at NASA-Ames Research Center and B. Barber at the California Institute of Technology for technical assistance. This research was supported in part by NASA grant NAGW1941, National Science Foundation grant number CTS-8813006, and the California Institute of Technology. Contribution \# 4870, Division of Geological and Planetary Sciences.

\section{@ American Astronomical Society • Provided by the NASA Astrophysics Data System}




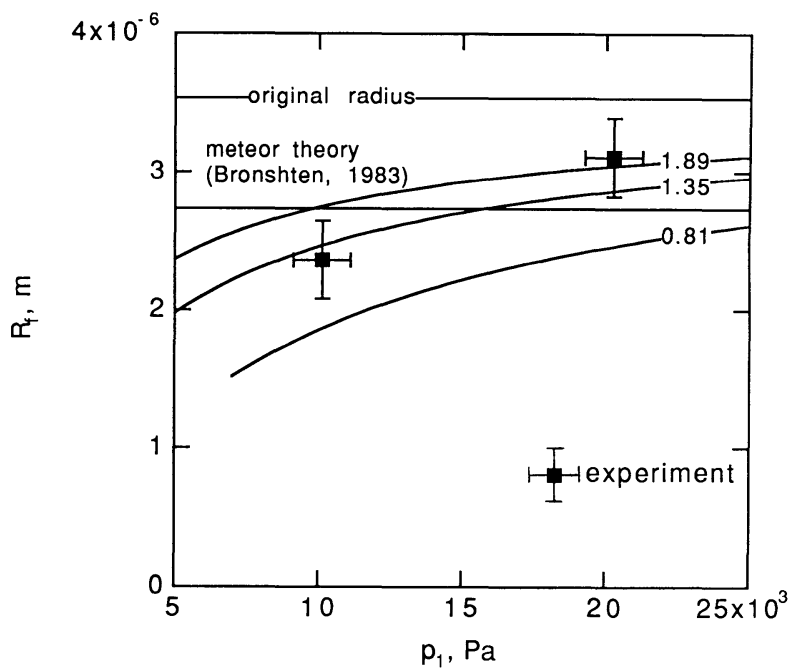

FIG. 11--Ablated radius $R_{f}$ for xenon at $295 \mathrm{~K}$ vs. pressure $p_{1}$ for various $Q\left(\mathrm{MJ} \mathrm{kg}^{-1}\right)\left(\rho_{p}=2420 \mathrm{~kg} \mathrm{~m}^{-3}\right.$ and $\left.v_{0}=4500 \mathrm{~m} \mathrm{~s}^{-1}\right)$. The two experimental values of $v_{0}$ were of slightly different value $\left(4540 \mathrm{~m} \mathrm{~s}^{-1}\right.$ for $p_{1}=0.1 \mathrm{~atm}$ and $4390 \mathrm{~m} \mathrm{~s}^{-1}$ for $p_{1}=0.2 \mathrm{~atm}$ ). The meteor theory curve is calculated assuming no variation of $\sigma$ with gas pressure. There is agreement between the present model and the experiment results for $Q=1.5 \pm 0.2 \mathrm{MJ} \mathrm{kg}^{-1}$.

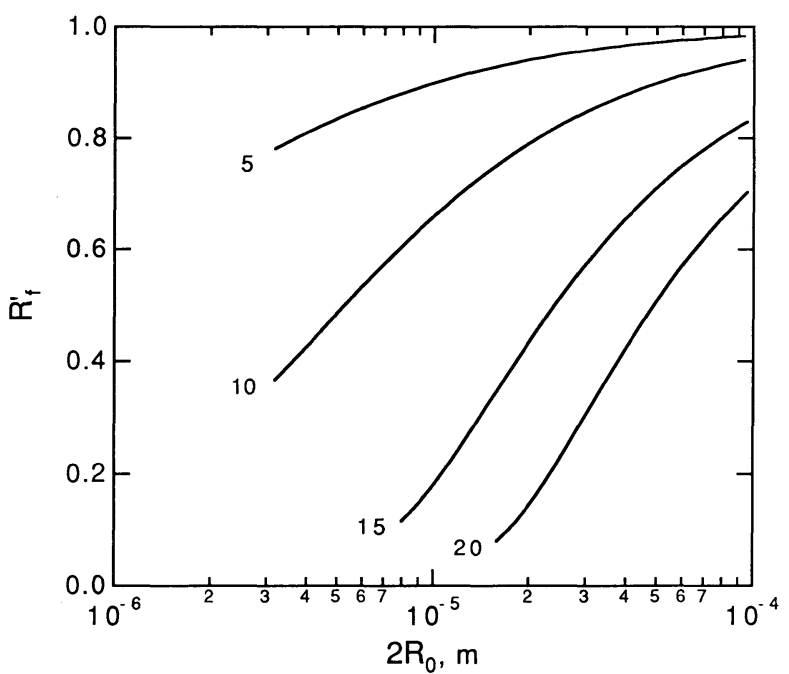

Fig. 13. $-R_{f}^{\prime}$ vs. $2 R_{0}$ for various values of $v_{0}\left(\mathrm{~km} \mathrm{~s}^{-1}\right)\left(Q=1.9 \mathrm{MJ} \mathrm{kg}^{-1}\right.$, $\left.\rho_{p}=1000 \mathrm{~kg} \mathrm{~m}^{-3}\right)$. The curves are terminated on the left-hand side where the condition that a bow shock forms ahead of the particle (i.e., $\mathrm{Kn}_{1} \leq 0.33$ ) is violated.

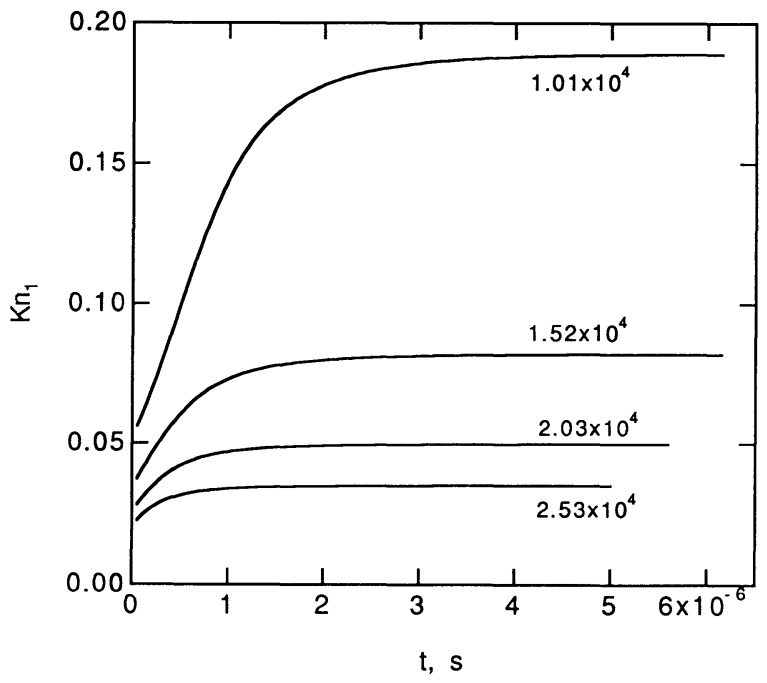

FIG. 12.-Free stream Knudsen number $\mathrm{Kn}_{1}$ histories for particles ablating in various free stream gas pressures $(\mathrm{Pa})\left(Q=1.9 \mathrm{MJ} \mathrm{kg}^{-1}, \rho_{p}=10^{3} \mathrm{~kg} \mathrm{~m}^{-3}\right.$, $v_{0}=10 \mathrm{~km} \mathrm{~s}^{-1}$, and $R_{0}=5$ microns).

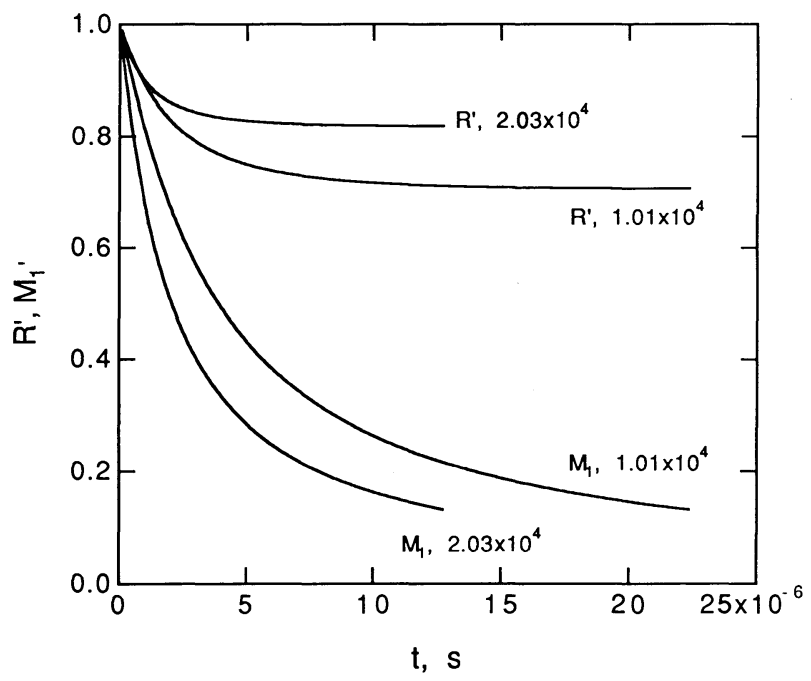

FIG. 14. $-M^{\prime}$ and $R^{\prime}$ vs. $t$ for two pressures $(\mathrm{Pa})\left(Q=1.35 \mathrm{MJ} \mathrm{kg}^{-1}, \rho_{p}=\right.$ $2420 \mathrm{~kg} \mathrm{~m}^{-3}, v_{0}=4.5 \mathrm{~km} \mathrm{~s}^{-1}$, and $R_{0}=3.53$ microns).

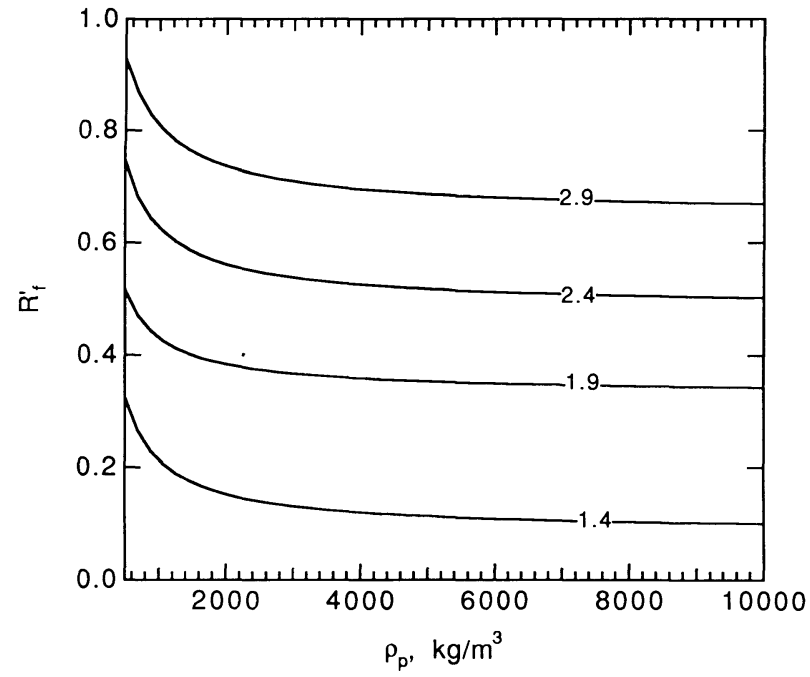

FIG. $15 .-R_{f}^{\prime}$ vs. $\rho_{p}$ for various $Q\left(\mathrm{MJ} \mathrm{kg}^{-1}\right)\left(v_{0}=10 \mathrm{~km} \mathrm{~s}^{-1}, R_{0}=5\right.$ microns, and $\left.p_{1}=0.2 \mathrm{~atm}\right)$. 


\section{REFERENCES}

Abramowitz, M., \& Stegun, J. E. 1970, Handbook of Mathematical Functions (Washington, DC: GPO)

Bansal, N. P. \& Doremus, R. H. 1986, Handbook of Glass Properties (New York: Academic Press)

IOI Becker, D. G. \& Slattery, J. C. 1973, ApJ, 186, 1127

IfI Becker, D. G., \& Friichtenicht, J. F. 1971, ApJ, 166, 699

Bronshten, V. A. 1983, Physics of Meteoric Phenomena (Boston: Reidel)

Crowe, C. T. 1967, AIAA J., 5, 1021

Eckert, E. R. G., \& Drake, R. M. 1972, Analysis of Heat and Mass Transfer (New York: McGraw Hill)

Forney, L. J., Walker, A. E., \& McGregor, W. K. 1987, Aero. Sci. \& Tech., 6, 143
Friichtenicht, J. F., \& Becker, D. G. 1971, ApJ, 166, 717

Friichtenicht, J. F., Slattery, J. C., \& Tagliaferri, E. 1968, ApJ, 151, 747

Hirschfelder, J., Curtiss, C., \& Bird, R. 1964, Molecular Theory of Gases and Liquids (New York: Wiley)

Liepmann, H. W., \& Roshko, A. 1957, Elements of Gasdynamics (New York: Wiley)

Rulison, A. J., Flagan, R. C., \& Ahrens, T. J. 1990, Rev. Sci. Instr., in press

Sheckler, C. A., \& Dinger, D. R. 1990, J. Am. Ceram. Soc., 73(1), 24

Slattery, J. C., \& Friichtenicht, J. F. 1967, ApJ, 147, 235 